\title{
Synthesis of Mesoporous Silica Microsphere from Dual Surfactant
}

\author{
Venkatathri Narayanan* \\ Department of Chemistry, National Institute of Technology, \\ Warangal 506 004, Andhra Pradesh, India
}

Received: June 9, 2008; Revised December 10, 2008

\begin{abstract}
A new procedure is reported to synthesis mesoporous silica micro sphere for the first time. In these method two surfactants namely Span 80 and Tween 80 were used. Small angle X ray diffraction and N2 adsorption analysis shows the synthesized material has mesoporous property. The material has spherical morphology with 1-10 $\mu \mathrm{m}$ particle size. Beside the material found to have microcapsule property as observed from the Transmission electron microscopy. The Fourier transform Infrared spectroscopic analysis reveals that the materials are similar to other mesoporous materials. We also encapsulated an UV-absorber Ibuprofen inside the microcapsule, by mixing it before the synthesis. This shows a possibility of the materials in cosmetic applications.
\end{abstract}

Keywords: mesoporous silica micro sphere, Tween 80 , Span 80

\section{Introduction}

Since silica based mesoporous molecular sieves using surfactant assemblies as supramolecular templates were reported in $1992^{1,2}$, mesoporous materials have opened many new possibilities for application in catalysis ${ }^{3}$, separation ${ }^{4}$, and nanoscience due to their tunable pore sizes and very large surface $\operatorname{areas}^{5-7}$. More recently, mesoporous silica with morphologies including thin films, monoliths, hexagonal prisms, toroids, discoids, spirals, dodecahedron and hollow tubular shapes have been synthesized ${ }^{8-12}$. In conventional procedures, silica spheres have been obtained using surfactants, block copolymers and recently by using colloidal suspensions ${ }^{13}$. Normally polymers are used in synthesis of hollow sphere. Only few are reported in surfactants. Tween 80 and Span 80 are commercially available surfactants. Preparation of micro sized silica spheres using Tween 80 and Span 80 surfactants have never been reported. The Tween 80 's chemical name is Poly oxy ethylene sorbitan mono oleate or Sorbitan monooleate ethoxylate and Span 80's chemical name is Sorbitan oleate or Sorbitan (Z)- mono 9-octadecenoate. Their densities are 1.064 (Tween 80) and 0.986 (Span 80). They are high hydrophilic - lipophilic balance (HLB) and low HLB surfactants respectively ${ }^{14}$. They are used to control the viscosity and increase the Water-1/Oil/Water-2 emulsion structure.

This paper described a novel synthesis pathway for mesoporous silica microspheres of 1-10 um particle size using dual surfactant. The synthesized material was characterized by various novel techniques and its storage property of a UV-absorber is studied for cosmetic applications.

\section{Experimental}

All chemicals used in this work were commercially available and were used without further purification. $\mathrm{Na}_{2} \mathrm{SiO}_{3}$ (Samchun pure chemical co. Ltd, South Korea), Span 80 (Daejung Chemicals \& metals co Ltd., South Korea), Tween 80 (Shinyo pure chemicals co. Ltd., Japan), $\mathrm{NH}_{4} \mathrm{HCO}_{3}$ (95\%, Dae-Jung chemicals and metals co. Ltd, South Korea), n-hexane (95\%, Dae-Jung chemicals and metals co. Ltd., South Korea).

Mesoporous silica microsphere was obtained by interfacial reaction. There are three solutions namely, sodium silicate solution,
Surfactants (Tween 80 and Span 80) and n-hexane mixture and ammonium bicarbonate solution were used in the present synthesis. The first solution was prepared by mixing distilled water and sodium silicate $(29.9 \mathrm{~g})$ (4 $\mathrm{M}$ aqueous solution $(36 \mathrm{~mL})$ ). Second mixture was prepared by mixing n-hexane $(72 \mathrm{~mL})$, Tween $80(1.0 \mathrm{~g})$ and Span $80(0.50 \mathrm{~g})$ for the stabilization of the water/oil emulsion. The last solution was made by mixing Distilled water and precipitant $\left(\mathrm{NH}_{4} \mathrm{HCO}_{3}, 9.8 \mathrm{~g}\right)(2 \mathrm{M}$ aqueous solution $(252 \mathrm{~mL}))$. First, n-hexane and surfactants were mixed by using a homogenizer (Matsushita Electric Industrial Co. Ltd., Japan, and Model No. National SSC811EA with $1000 \mathrm{rpm}$ ). To this solution, sodium silicate solution was added. After being emulsified for 1 minute, this mixture was poured into ammonium bicarbonate solution with stirring. After a thorough mixing, a white precipitate was formed. The mixture was stirred for another 2 hours to continue precipitation at constant temperature (298 K). The solid was filtered and washed with fresh distilled water for several times. Finally, the solid was dried at $373 \mathrm{~K}$ for 12 hours.

$\mathrm{X}$ ray diffractograms were recorded on Rigaku Multiplex diffractometer using $\mathrm{Cu} \mathrm{K} \alpha$ radiation and a proportional counter as detector. A divergence slit of 1/328 on the primary optics and an anti-scatter slit of $1 / 168$ on the secondary optics were employed to measure data in the low angle region. The scanning electron micrographs were taken using a Topcon, SM-300. The samples were deposited on a sample holder with an adhesive carbon foil and sputtered with gold. For transmission electron microscopy, the samples were ground and deposited on a circular disc of fine copper mesh covered with collodion. Images were taken using a JEOL JSM-2000 EX electron microscope operated at an acceleration voltage of $200 \mathrm{kV}$. The specific surface area (BET) of the samples was determined using a Micromeritics ASAP 2010 volumetric adsorption analyzer. Before $\mathrm{N}_{2}$ adsorption samples was evacuated in vacuum at $573 \mathrm{~K}$. The data points of $\mathrm{p} / \mathrm{p}_{0}$ in the range of about $0.05-0.3$ were used in the calculations. The FT-IR spectra in the framework region were recorded in the diffuse reflectance mode (Nicolet 60SXB) using 1:300 ratio of sample with $\mathrm{KBr}$, pellet. UV-spectra were analyzed using JASCO -V-530, UV-Vis spectrophotometer. 


\section{Results and Discussion}

Figure 1 illustrates the low angle $\mathrm{X}$ ray diffraction (XRD) patterns of the as-synthesized sample. The calcined sample pattern does not vary considerably, however a small peak broadening is observed. The high intensed peak at $2 \theta=1.25^{\circ}$ shows it is a mesoporous material ${ }^{15}$.

The Scanning electon microscopic image of the material posse's spheroid and the particle size is in between 1 to $10 \mu \mathrm{m}$ (Figure 2a). The sample synthesized without surfactants namely, Tween 80 and Span 80 shows irregular flakes morphology (Figure 2c). The Transmission electron microscopic picture shows presence of microcapsule property (Figure 2b). The wall thickness of the shell was found to be $0.25 \mu \mathrm{m}$ and the diameter of the silica particle is about $1.5 \mu \mathrm{m}$. Therefore the shell wall was considerably thin, and its thickness was estimated to be about $20 \%$ of the total size of the particle, although most of the other reported silica hollow sphere has thicker shell walls. Similar reports are available in the literature ${ }^{15}$. The sample synthe-

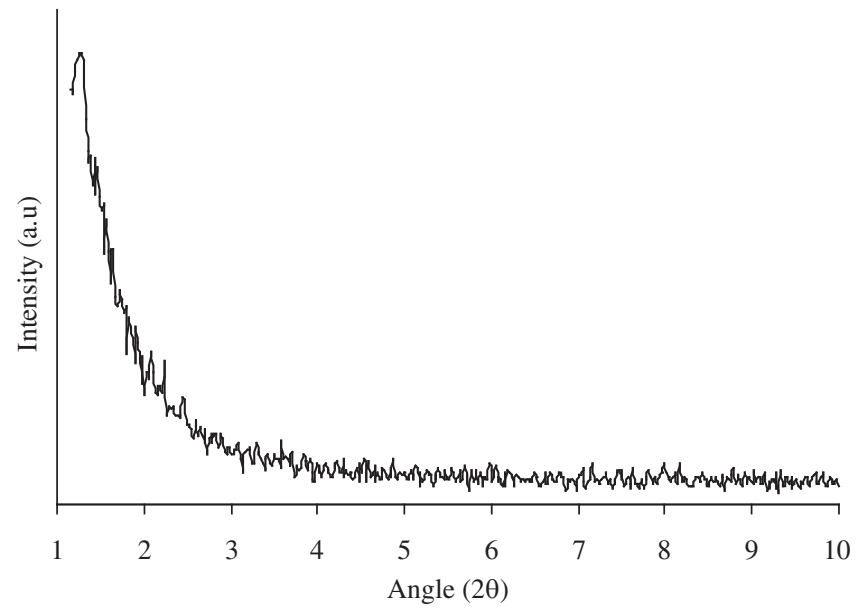

Figure 1. X ray diffraction pattern of as synthesized sample prepared from Tween 80 and Span 80 surfactant mixture.

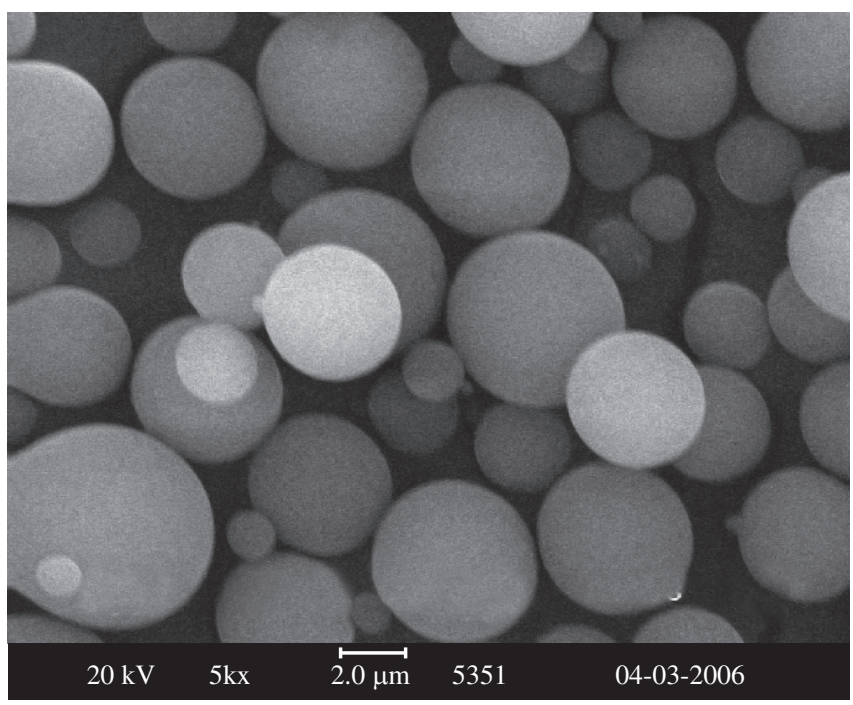

(a)

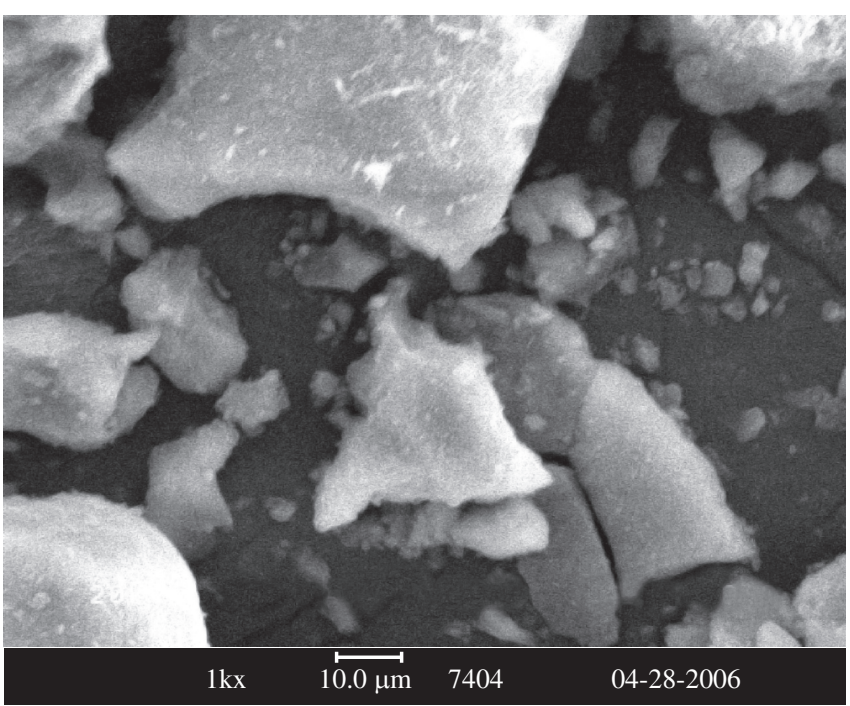

(c)

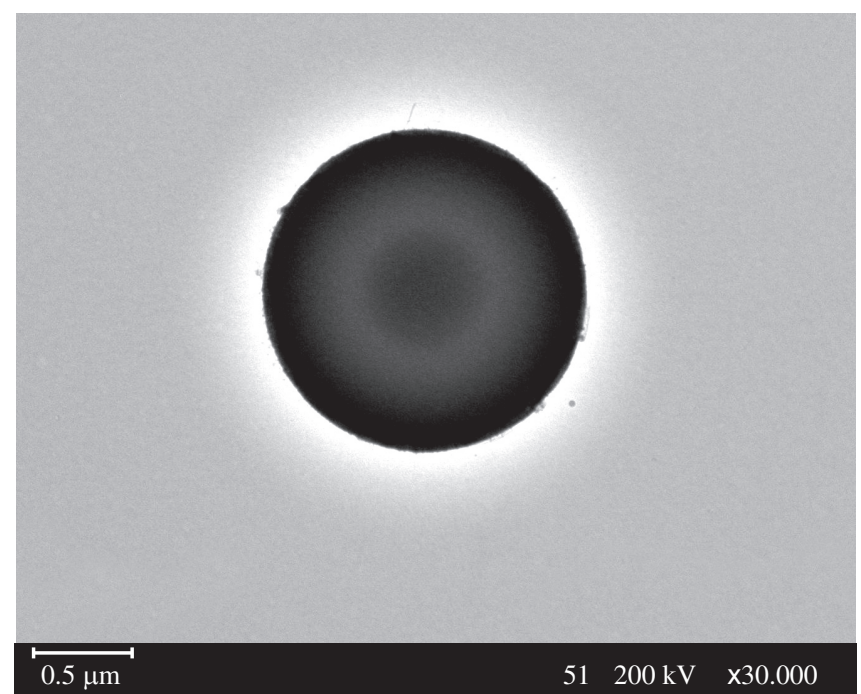

(b)

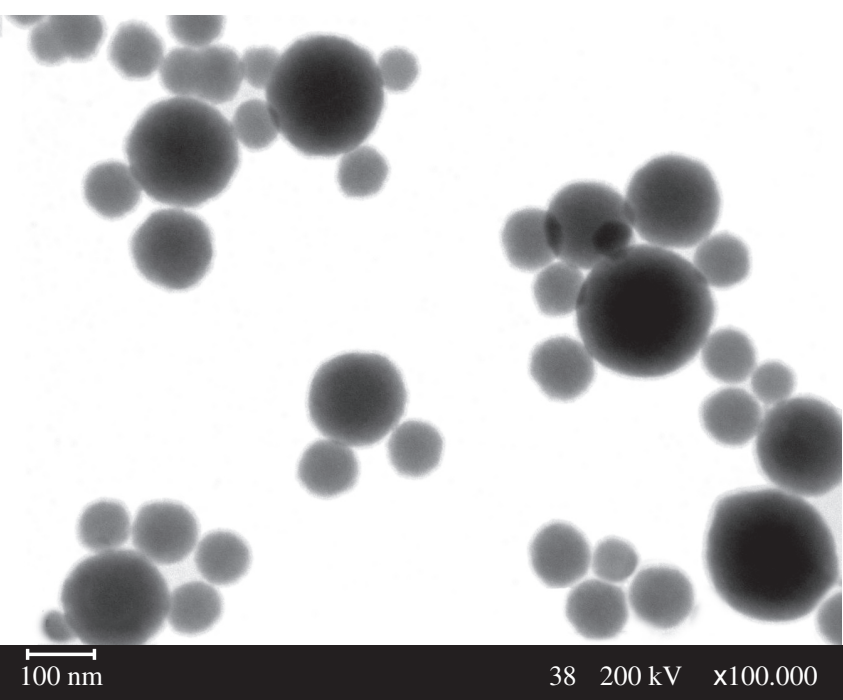

(d)

Figure 2. Scanning electron microscopic photograph of sample prepared from a) Tween 80 and Span 80 surfactant mixture and c) without surfactant and Transmission electron microscopic photograph of sample prepared from b) Tween 80 and Span 80 surfactant mixture and d) Tween 80/Span 80 surfactant. 
sized with any one of the surfactant give nanosphere, but the hollow sphere property lost (Figure 2d). The physicochemical nature of the two surfactants in combine plays a major role in hollow formation. Yield was $99 \%$ with respect to the theoretical yield of precipitated silica. The total weight of silica $\left(\mathrm{SiO}_{2}\right)$ present in whole sodium silicate was taken as the theoretical yield. This silica microcapsule was not mechanically fragile. When this microcapsule was pounded hard in a mortar, no parts of the microcapsule were broken. The thermo gravimetric analysis shows that the sample loss about $10 \%$ of its total weight on calcinations. EDX analysis of the sample after calcinations indicates the presence of only silicon other than oxygen.

Typical nitrogen sorption isotherms at $77 \mathrm{~K}$ and the corresponding pore size distribution are shown in Figure 3. The nitrogen isotherms indicate a linear increase of the amount of adsorbed nitrogen at low pressures $(\mathrm{P} / \mathrm{Po}=0.35)$. The resulting isotherm can be classified as a type IV isotherm with a type $\mathrm{H} 2$ hysteresis, according to the IUPAC nomenclature ${ }^{15-18}$. The steep increase in nitrogen uptake at relative pressures in the range between $\mathrm{P} / \mathrm{Po}=0.40$ and 0.60 is reflected in a narrow pore size distribution. Thus, the variation of the catalyst in the solution during the growth process enables one to adjust and to control pore structural parameters such as the specific surface area $\left(900 \mathrm{~m}^{2} / \mathrm{g}\right)$, the specific pore volume $\left(1.29 \mathrm{~cm}^{3} / \mathrm{g}\right)$, and the average pore diameter $\left(31 \mathrm{~A}^{\circ}\right)$.

The FT-IR spectrum of as-synthesized sample (Figure 4) shows peaks around 1700 and $3430 \mathrm{~cm}^{-1}$ corresponding to the carboxyl and hydroxyl groups ${ }^{19}$ respectively. The adsorption peak belonging to the $\mathrm{Si}-\mathrm{O}$ stretching vibration of $\mathrm{Si}-\mathrm{OH}$ bond appears ${ }^{20}$ at $960 \mathrm{~cm}^{-1}$. The weak peaks at 2855 and $2920 \mathrm{~cm}^{-1}$ belong to the stretching vibrations of $\mathrm{C}-\mathrm{H}$ bonds, which show a few organic groups are adsorbed on the spheres. The strong peaks near 1100, 802 and $467 \mathrm{~cm}^{-1}$ agree to the Si-O-Si bond which implies the condensation of silicon source ${ }^{21}$.

Ibuprofen with the molecules size of $1.0 \times 0.6 \mathrm{~nm}$ was used to examine the UV-absorbing capacity ${ }^{22,23}$. The UV-absorber was introduced inside the shell by mixing prier to synthesis. Figure 5 shows the UV absorbance spectra of ibuprofen encapsulated silica micro capsule, pure ibuprofen and ibuprofen absorbed on silica microcapsule. Pure ibuprofen shows highest intensity followed by ibuprofen absorbed silica microcapsule. The UV absorbance intensity of ibuprofen encapsulated samples decrease marginally. This illustrates that the capsule could hold more UV-absorber molecules.

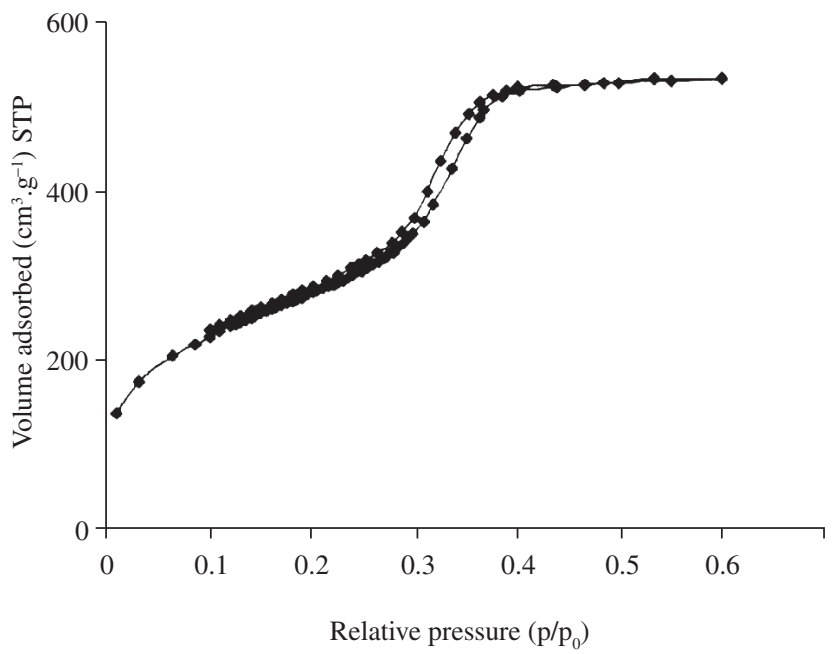

Figure 3. Nitrogen sorption isotherms at $77 \mathrm{~K}$ for calcined sample prepared from Tween 80 and Span 80 surfactant mixture.

\section{Conclusions}

A novel procedure brought out for synthesis of mesoporous silica microcapsule from dual surfactant. Mono and surfactant free synthesis leads different material. It can hold good number of an UV absorber say Ibuprofen by presynthesis modifications. It can be used in cosmetics applications.

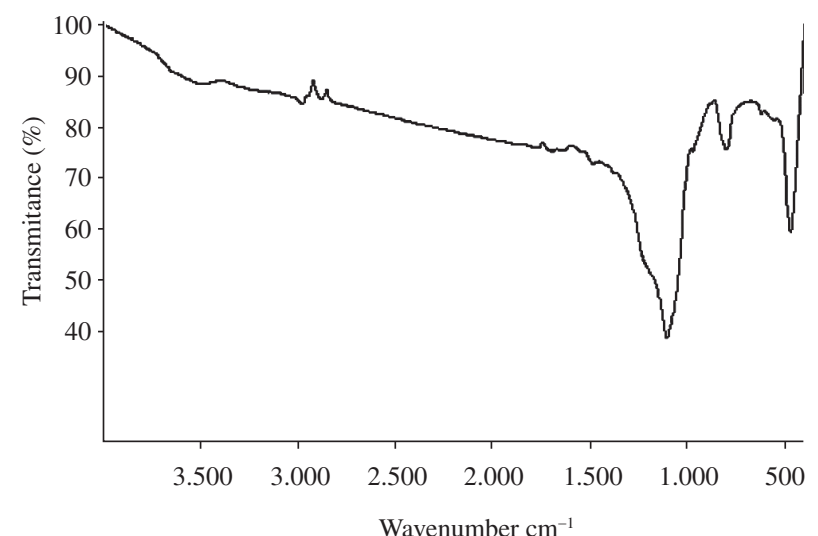

Figure 4. Fourier transform Infrared spectroscopic analysis of the as-synthesized sample prepared from Tween 80 and Span 80 surfactant mixture, in the framework region $($ Bands $=467(\mathrm{~s}), 802(\mathrm{~m}), 960(\mathrm{vw}), 1100(\mathrm{vs}), 1700(\mathrm{vw})$, $2855(w), 2920(w)$ and $3430(w))$.

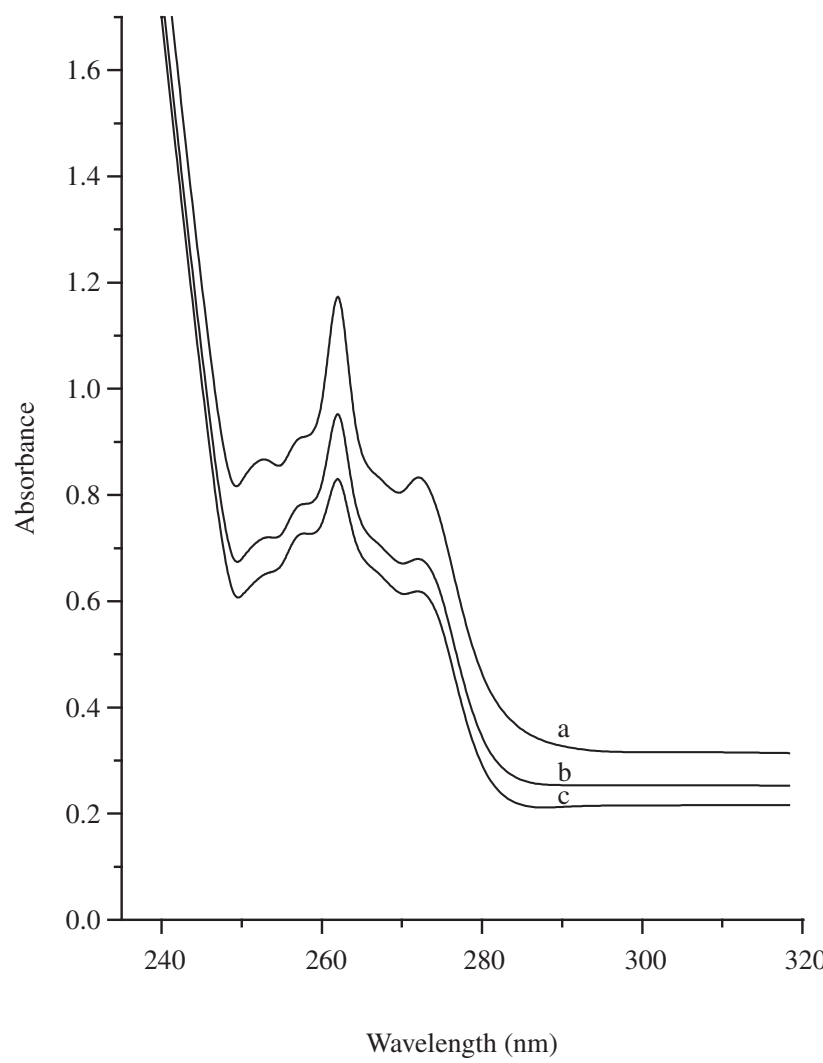

Figure 5. UV-Vis spectra of a) pure Ibuprofen, b) Ibuprofen dispersed silica microsphere and c) Ibuprofen encapsulated silica microsphere prepared from Tween 80 and Span 80 surfactant mixture. 


\section{References}

1. Beck JS, Vartuli JC, Roth WJ, Leonawicz ME, Kresge CT. A new family of mesoporous molecular sieves prepared with liquid crystal templates. J Am Chem Soc 1992; 114(27): 10834-10843.

2. Kresge CT, Leonowicz ME, Roth WJ, Vartuli JC, Beck JS. Ordered mesoporous molecular sieves synthesized by a liquid crystal template mechanism. Nature 1992; 359(1): 710-712.

3. Tanev PT, Chibwe M, Pinnavaia TJ. Titanium - containing mesoporous molecular sieves for catalytic oxidation of aromatic compounds. Nature 1994; 368(1): 321-323.

4. Llewellyn PL, Ciesla U, Decher H. MCM-41 and related materials as media for controlled polymerization process. Stud Surf Sci Catal 1994; 84(3): 2013-2018.

5. Wu CG, Bein T. Polyaniline wires in oxidant - containing Mesoporous channel Hosts. Chem Mater 1994; 6(8): 1109-1112.

6. Agger JR, Anderson MW, Pemble ME, Growth of Quantum - Confined Indium phosphide inside MCM-41. J. Phys Chem B 1998; 102(18): 3345-3353.

7. Corma A. In organic Solid Acids and Their use in Acid - Catalysed Hydrocarbon reactions. Chem Rev 1995; 95(1): 559-614.

8. Yong H, Coombs N, Ozin GA. Morphogenesis of shapes and surface patterns in Mesoporous silica. Nature 1997; 386(1): 692-695.

9. Trau M, Yao N, Kim E, Xia Y, Whitesides GA, Aksay IA. Microscopic patterning of oriented mesoscopic silica through guided growth. Nature 1997; 390(1): 674-676.

10. Lin HP, Mou CY. "Tubules - with in - a - Tubule" Hierarchical order of Mesoporous Molecular sieves in MCM-41. Science 1996; 273(1): 765-768.

11. Schacht S, Hao Q, Voigt IG, Stucky GD, Schuth F. Oil - water Interface Templating of Mesoporous Macroscale Structures. Science 1996; 273(1): 768-771.
12. Oliver S, Kuperman A, Coombs N, Lough A, Ozin GA. Lamellar aluminophosphates with surface patterns that mimic diatom and radiolarian microskeletons. Nature 1995; 378(1): 47-50.

13. Stein A. Sphere templating methods for periodic porous solids. Micropor. Mesopor. Mater 2001; 44-45(1): 227-39.

14. Fortes MA, Fatima Vaz M. Unusual properties of foams at low pressures. J Colloid Interface Sci 2003; 266(1): 202-207.

15. Fujiwara M, Shiokawa K, Tanaka Y, Nakahara Y. Preparation and Formation mechanism of silica Microcapsules (Hollow sphere) by water/ oil/water Interfacial reaction. Chem Mater 2004; 16(25): 5420-5426.

16. Brunauer S, Deming LS, Deming WS, Teller E. On a theory of the vander waals Adsorption of Gases. J Am Chem Soc 1940; 62(7): 1723-1732.

17. De Boer JH. The Structure and Properties of Porous Materials. London: Butterworths; 1958.

18. INTERNATIONAL UNION OF PURE AND APPLIED CHEMISTRY. Reporting Physisorption Data for Gas/Solid Systems. Pure Appl Chem 1957; 87(1): 603-608.

19. Li Y, Xu C, Wei B, Zhang X, Zheng M, Wu D, Ajayan PM. Self organized Ribbons of Aligned carbon Nano tubes. Chem Mater 2002; 14(2): 483-485.

20. Shan Y, Gao L, Zheng S. A facile approach to load Cd Se nanocrystallites into mesoporous SBA - 15. Mater Chem Phys 2004; 88(1): 192-6.

21. Srinivasu P, Anand C, Alam S, Ariga K, Halligudi SB, Balasubramanian VV, A. Vinu A. Direct synthesis and the morphological control of Highly ordered Two - Dimensional P6mm Meoporous Niobium silicates with High Niobium content. J Phys Chem C 2008; 112(27): 10130-10140.

22. Zhu Y, Shi J, Chen H, Shen W, Dong X. A facile method to synthesize novel hollow mesoporous silica spheres and advanced storage property. Micropor Mesopor Mat 2005; 84(1-3): 218-222.

23. Chen JF, Ding HM, Wang JX, Shao L. Preparation and characterization of porous hollow silica nanoparticles of drug delivery application. Biomaterials 2004; 25(4): 723-727 